\title{
SPACE-CHARGE EFFECTS IN IONIZATION BEAM PROFILE MONITORS *
}

\author{
V. Shiltsev $^{\dagger}$, Fermilab, PO Box 500, MS339, Batavia, IL, USA 60510
}

\begin{abstract}
Ionization profile monitors (IPMs) are widely used in accelerators for non-destructive and fast diagnostics of high energy particle beams. At high beam intensities, the spacecharge forces make the measured IPM profiles significantly different from those of the beams. We analyze dynamics of the secondaries in IPMs and develop an effective algorithm to reconstruct the beam sizes from the measured IPM profiles. Efficiency of the developed theory is illustrated in application to the Fermilab $8 \mathrm{GeV}$ proton Booster IPMs.
\end{abstract}

\section{INTRODUCTION}

Particle accelerators heavily rely on precise diagnostics and control of critical beam parameters such as intensity, pulse structure, position, transverse and longitudinal beam sizes, halo, etc [1]. Ionization profile monitors (IPMs) [2-7] are fast and non-destructive diagnostic tools used in proton and ion linacs, colliders and rapid cycling synchrotrons (RCS) [8-10]. They operate by collecting ions or electrons created after the ionization of residual vacuum molecules by high energy charged particle beams $[1,11]$, which are then guided to a detector by a uniform external electric field $E_{\text {ext }}$. The detector is usually made of many thin parallel strips, whose individual signals are registered to make the beam profile signal ready for processing - see Fig.1.

Space-charge forces of the primary beams make the measured IPM profiles different from those of the beams and must be correctly accounted. Brute force numerical modeling $[12,13]$ can successfully reproduce experimentally measured IPM profiles, but offer limited predictive physics insights. Several phenomenological fits were proposed to relate the measured beam size $\sigma_{m}$ and the initial beam size $\sigma_{0}$ - see, e.g., Refs. [3, 12, 14, 15] - but despite acceptable data approximation, they are not based on clear physical reasons for as many four free parameters and exponents. Below we present an effective algorithm [16] to reconstruct the beam sizes from measured IPM profiles and known key parameters, such as high-energy beam intensity $N$ and IPM extracting field $E_{\text {ext }}=V_{0} / D$ is the guiding electric field due to the voltage gradient $v_{0}$ across the IPM gap $D$.

\section{SPACE-CHARGE DRIVEN IPM PROFILE EXPANSION}

The general equations of transverse motion of the nonrelativistic ions born in the IPM in the acts of ionization of the residual gas molecules are $x^{\prime \prime}(t)=\frac{Z e}{M} E_{\text {ext }}+$

\footnotetext{
* This manuscript has been authored by Fermi Research Alliance, LLC under Contract No. DE-AC02-07CH11359 with the U.S. Department of Energy, Office of Science, Office of High Energy Physics.

$\dagger$ shiltsev@fnal.gov
}

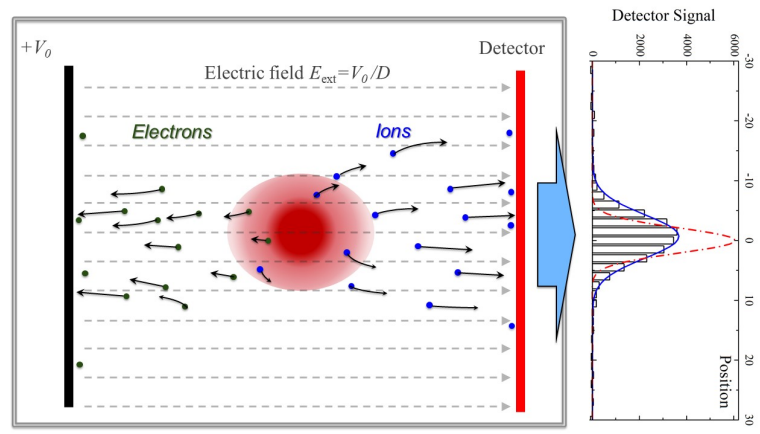

Figure 1: Transverse cross-section of a high energy beam (red) in vertical IPM and schematically shown motion of secondary ions (blue dots) and electrons (green dots) under the impact of horizontal extracting electric field $E_{\text {ext }}$ and space-charge field of the primary beam. The diagram on the right shows the IPM detector signals at right before extraction of an intense beam of $N=4.6 \cdot 10^{12}$ protons from the Fermilab Booster synchrotron. The actual rms proton vertical size of the proton beam is $\sigma_{0}=2.1 \mathrm{~mm}$ - see dashed red curve, while the rms width of the IPM signal is $\sigma_{m}=3.6$ $\mathrm{mm}$, see blue line for the Gaussian fit.

$\frac{Z e}{M} E_{x}^{\mathrm{SC}}(x, y, t)$ and $y^{\prime \prime}(t)=\frac{Z e}{M} E_{y}^{\mathrm{SC}}(x, y, t)$, where $Z e$ and $M$ are the ion's charge and mass, $E_{\text {ext }}=V_{0} / D$ is the IPM extracting external electric field which is assumed here to be horizontal and generated by application of high voltage $V_{0}$ over the gap $D$. The electric force of the primary Gaussian beam is $E_{(x, y)}^{\mathrm{SC}}=\frac{2 J(t)}{v_{p}} \frac{(x, y)}{r^{2}}\left(1-\exp \left(-\frac{r^{2}}{2 \sigma_{0}^{2}}\right)\right)$, where $J(t)$ and $\sigma_{0}$ are the high energy beam current and rms transverse size, respectively, $v_{p}$ is its velocity, and $r^{2}=x(t)^{2}+y(t)^{2}$. The particle's transverse velocity $y^{\prime}(t)$ is mostly accumulated while it is passing through the beam core area $r \lesssim \sigma_{0}$, while its trajectory outside the core is mostly ballistic and scales approximately linearly with time until the particle reaches the IPM detector plane at $t=\tau_{2}=\sqrt{\frac{2 M d}{Z e E_{\text {ext }}}}$, here $d$ is the average distance from the beam center to the detector. IPMs usually operate with electric fields $E_{\text {ext }} \sim O(100-1000 \mathrm{~V} / \mathrm{mm})$ which significantly exceed the space-charge field $E^{\mathrm{SC}} \sim O(1-10 \mathrm{~V} / \mathrm{mm})$ and that makes the equation of motion in the $x$-plane trivial $x(t) \approx \frac{Z e E_{\mathrm{ext}}}{2 M} t^{2}$. For slow varying or DC proton current $J(t)=J$, solution of the equation of motion inside the beam, $t \lesssim \tau_{0}$, is $y(t) \approx y_{0} \operatorname{ch}\left(t / \tau_{1}\right)+v_{0, y} \tau_{1} \operatorname{sh}\left(t / \tau_{1}\right)$, where we introduce a characteristic expansion time due to the space-charge $\tau_{1}=\left(\frac{e Z U_{S C}}{M \sigma_{0}^{2}}\right)^{-1 / 2}$, and the space-charge potential $U_{S C}=J /\left(4 \pi \epsilon_{0} v_{p}\right) \approx 30[\mathrm{~V} / \mathrm{A}] J / \beta_{p}, \beta_{p}=v_{p} / c$, $v_{p}$ is the main (proton) beam velocity, $c$ is the speed of light, and $\epsilon_{0}$ is the permittivity of vacuum [17]. A charac- 
teristic time for the secondaries to get extracted out of the beam by the external electric field $E_{\text {ext }}=V_{0} / D$.Eq.(??) is $\tau_{0}=\sqrt{\frac{2 M \sigma_{0}}{Z e E_{\text {ext }}}}=\tau_{2} \sqrt{\frac{\sigma_{0}}{d}}$.

The major part of the ion trajectory lays outside the beam and, therefore, $r(t) \approx x(t)$ and, given that the initial coordinates are small compared to the average distance $d$ from the beam center to the IPM detector plane $\left(x_{0}, y_{0}\right) \ll d \approx D / 2$, we have for $y$ plane $y^{\prime \prime}(t)=\frac{y}{\tau_{1}^{2}} \frac{2 \sigma_{0}^{2}}{r^{2}(t)}\left(1-\exp \left(-\frac{r^{2}(t)}{2 \sigma_{0}^{2}}\right)\right)$. The solution of this second-order ordinary differential equation can be obtained assuming $\tau_{0} / \tau_{1} \leq 1, y_{[1]}(t)=y_{0} \cdot[1+$ $\frac{\tau_{0}^{2}}{\tau_{1}^{2}}\left(\frac{t}{3 \tau_{0}}\left(\Gamma\left(\frac{1}{4}\right)-\Gamma\left(\frac{1}{4}, \frac{t^{4}}{\tau_{0}^{4}}\right)\right)-\frac{1}{2} \sqrt{\pi} \operatorname{erf}\left(\frac{t^{2}}{\tau_{0}^{2}}\right)+\frac{\tau_{0}^{2}}{6 t^{2}}\left(1-\exp \left(-\frac{t^{4}}{\tau_{0}^{4}}\right)\right)\right]$ Here $\Gamma(a)$ and $\Gamma(a, x)$ are complete and incomplete gamma functions related as $\Gamma(a)=\Gamma(a, 0)$. This equation is linear with respect to $y_{0}$, therefore, the space-charge expansion in IPM results in proportional magnification of the profile of the distribution of the secondary particles. Accordingly, the rms transverse size of the IPM profile at the time when the secondary particle reaches the IPM detector $t=\tau_{2} \gg \tau_{0}$ becomes:

$$
\sigma_{m}=\sigma_{0} \cdot h \approx \sigma_{0} \cdot\left[1+\frac{2 U_{S C}}{E_{\mathrm{ext}} \sigma_{0}}\left(\frac{\Gamma\left(\frac{1}{4}\right)}{3} \sqrt{\frac{d}{\sigma_{0}}}-\frac{\sqrt{\pi}}{2}\right)\right] .
$$

The gamma-function $\Gamma\left(\frac{1}{4}\right) \approx 3.625$. The space-charge expansion factor $h$ is determined only by the space-charge potential $U_{S C}$, the primary beam size $\sigma_{0}$, the IPM extracting field $E_{\text {ext }}=V_{0} / D$, and the beam-to-MCP distance $d$ but it does not depend on the type of secondary species (their mass and charge, etc). Eq.(1) can be easily solved, and the original $\sigma_{0}$ can be found from $\sigma_{m}$ with other IPM and beam parameters known [16].

A similar analysis for uniform, rather than Gaussian, primary proton beam current density distribution with radius $a$ results in an exact solution in elementary functions that is very close to Eq. $(1)$, with the numerical factor $2 \Gamma(1 / 4) / 3 \approx$ 2.42 replaced by $(4 \sqrt{2} / 3) \approx 1.88$, and substitution of equivalent $\sigma_{0}=a / 2$ - see Ref. [16].

The effect of the high energy beam size aspect ratio $R=$ $\sigma_{x} / \sigma_{y}$ is relatively weak, too. Indeed, the space-charge factor $1 / \tau_{1}^{2}$ scales as $2 /(1+R)$ while the characteristic time $\tau_{0} \propto \sqrt{R}$. Therefore, the product $\tau_{0} / \tau_{1}^{2}-$ the second term in Eq.(1) - scales as $2 \sqrt{R} /(1+R)$. The latter is relatively small, i.e, 0.94 for $R=0.5$, and can be safely neglected for most common cases of $h \leq 2$.

To account for initial velocities of the secondaries $v_{0, y}$, one can assume them to be random with the rms value of $\sqrt{2 \mathcal{E}_{i} / M}$ and get in quadrature addition to Eq.(1):

$$
\sigma_{m}^{2}=\sigma_{0}^{2} h^{2}\left(U_{S C}, \sigma_{0}, E_{\mathrm{ext}}, d\right)+\left(\frac{4 \mathcal{E}_{i} d}{Z e E_{\mathrm{ext}}}\right) .
$$

The effect of the high-energy beam current $J(t)$ time structure, such as in bunched beams, depends on the rms bunch length $\tau_{b}$ and time between bunches $t_{b}$ and can be approximated by substitution $U_{S C} \rightarrow U_{S C}\left(1+t_{b} / \tau_{0}\right)$. See [16] for details as well analysis of the extreme case of short and rare bunches $\tau_{b} \ll\left(\tau_{0}, \tau_{1}, \tau_{2}\right) \ll t_{b}$.

The types of IPMs are distinguished by the species they collect - electrons or ions. The initial kinetic energy $\mathcal{E}_{i}$ for ionization electrons is about $35 \mathrm{eV}$ needed on average for ion-electron pair production by protons in hydrogen [18]. Corresponding smearing Eq.(2) of the particle position measured by the IPM is about $\sigma_{T}=D \sqrt{2 \mathcal{E}_{i} / Z e V_{0}}$, that is some $6 \mathrm{~mm}$ for a typical gap $D=100 \mathrm{~mm}$ and voltages as high as $V_{0}=20 \mathrm{kV}$. That is absolutely unacceptable for millimeter-scale or smaller primary beam sizes and the electron-collecting IPMs usually have to use a focusing external magnetic field $B_{x}$, parallel to the extracting electric field, to suppress the smearing. Physics principles, advantages and disadvantages of the IPMs with a magnetic field are discussed in [19].

As for ions, their initial kinetic energy is smaller depends on their kind and the type of reaction. For diatomic gases, the most relevant process is dissociative ionization by the primary fast protons, i.e., $p+\mathrm{H}_{2} \rightarrow p+H+H^{+}$with typical kinetic energy of the $\mathrm{H}^{+}$of the order of a few eV [20]. Corresponding smearing of the profile $\sigma_{T}$ in the ion-collecting IPMs is $O(1 \mathrm{~mm})$ [21]. These IPMs do not require external magnetic field and, therefore, are usually of smaller size, simpler design and lower cost. Two such monitors - vertical and horizontal- are installed in the Fermilab Booster rapid cycling synchrotron (RCS) and we apply our analysis to their experimentally measured profiles [22,23].

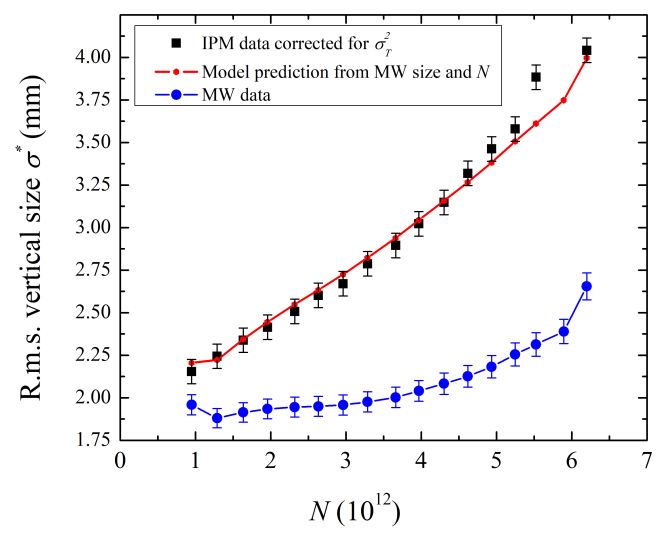

Figure 2: The Fermilab Booster IPM vertical rms beam size $\sigma^{*}$ right before beam extraction $\left(V_{0}=24 \mathrm{kV}, D=103 \mathrm{~mm}\right.$, black squares) $[16,22]$ vs the total proton beam intensity $N$. The theoretical predication of this paper's Eq.(1) (red line) is calculated using the initial beam sizes $\sigma_{0}$ as measured by the Multi-Wires emittance monitor (blue line). The measured IPM rms sizes $\sigma_{m}$ are corrected for the intensity independent smearing $\sigma^{*}=\sqrt{\sigma_{m}^{2}(N)-\sigma_{T}^{2}}$, with $\sigma_{T}^{2}=2.7 \mathrm{~mm}^{2}$. 


\section{APPLICATION FOR FERMILAB BOOSTER IPM}

The Fermilab Booster [24] is a $474.2 \mathrm{~m}$ circumference, alternating-gradient $15 \mathrm{~Hz}$ RCS accelerating protons from $0.4 \mathrm{GeV}$ at injection to $8.0 \mathrm{GeV}$ at extraction in $33.3 \mathrm{~ms}$, or about 20000 turns - half of the magnet cycle period. Correspondingly, all proton beam parameters (intensity, positions, bunch length, emittances) as well as accelerating RF frequencies and voltage significantly vary in the cycle. Typical total intensity of 84 circulating proton bunches is about $N=4.6 \cdot 10^{12}$.

The Booster proton beam dynamics is quite complex leading to the beam emittance growth and particle losses during the acceleration [22] which set limits on the high power operation of the entire Fermilab complex of accelerators for high energy neutrino physics $[25,26]$. Fast diagnostics of the Booster proton beam size is, therefore, of critical importance.

There are two types of instruments to measure beam sizes in the Booster - the Multi-Wires and IPMs. The Multi-Wires are intercepting devices installed in the Booster extraction beamline. When the proton beam strikes an individual wire (there are 48 wires spaced by $1 \mathrm{~mm}$ in each Multi-Wires), secondary electrons create a current in the wires which is amplified to produce the profile. The Multi-Wires beam size measurements data are presumed to be intensity independent and accurate to some 2-3\%. The IPMs operate in the ion collection mode and report the average rms beam sizes (determined by the Gaussian fits of the profiles) every turn.

Besides the space-charge expansion and the effect of the initial ion velocities Eq.(2), the IPM intensity independent profile smearing can be caused by a finite separation between the individual IPM charge collection strips, angular misalignment of the long and narrow strips with respect to the high energy beam trajectory, and by non-uniformity of the extraction electric field in the operational IPM aperture. All the above monitor-specific effects are independent of beam energy and can be taken into account either by detail simulations or via extensive test bench measurements or by cross-calibration of low intensity beam sizes measured by the IPM $\sigma_{m}$ and by the Multi-Wires $\sigma_{M W}$, or by other appropriate beam size monitors [1,9,22, 27-30]. In that case, the desired rms instrumental smearing can be found as $\sigma_{T}^{2}=\lim _{N \rightarrow 0}\left(\sigma_{m}^{2}(N)-\sigma_{M W}^{2}(N)\right)$. Comparison of the Booster IPM and Multi-Wires data at various beam intensities yields the intercept of $\sigma_{T}^{2}=2.8 \pm 0.1 \mathrm{~mm}^{2} \quad$ [22]

At high intensity, the average space-charge potential of the Booster proton beam is $U_{S C} \approx 18.2 \cdot N /\left(6 \cdot 10^{12}\right)$ [V]. Typical rms proton bunch length and bunch-to-bunch spacing are $\tau_{b} \approx 2-3 \mathrm{~ns}, t_{b} \approx 19 \mathrm{~ns}$. Characteristic times for the IPM with $D=103 \mathrm{~mm}$ and $V_{0}=24 \mathrm{kV}$ are $\tau_{b} \approx 2-3 \mathrm{~ns}$, $t_{b} \approx 19 \mathrm{~ns}, \tau_{0} \approx 22 \mathrm{~ns}, \tau_{1} \approx 67 \mathrm{~ns}$ (for $N=6 \cdot 10^{12}$ ) and $\tau_{2} \approx 110 \mathrm{~ns}$. Therefore, the beam profile expansion factor $h$ can be calculated by using Eq.(1) in which the original $\sigma_{0}$ is taken from the Multi-Wires data and with the beam-toMCP distance $d \approx D / 2=52 \mathrm{~mm}$. To take into account the time structure of the Booster bunched beam, the rms profile expansion coefficient $h$ Eq.(1) needs to be augmented by a numerical factor $\left[1+t_{b} / \tau_{0}\right]$.

$$
h=1+\frac{2 U_{S C}}{E_{\mathrm{ext}} \sigma_{0}}\left(\frac{\Gamma\left(\frac{1}{4}\right)}{3} \sqrt{\frac{d}{\sigma_{0}}}-\frac{\sqrt{\pi}}{2}\right) \cdot\left[1+t_{b} / \tau_{0}\right] .
$$

The resulting rms vertical IPM beam size estimates $h \sigma_{0}$ are found to be in excellent agreement with the measured IPM rms sizes $\sigma^{*}=\sqrt{\sigma_{m}^{2}(N)-\sigma_{T}^{2}}$ measured over a broad range of beam intensities as shown in Fig.2.

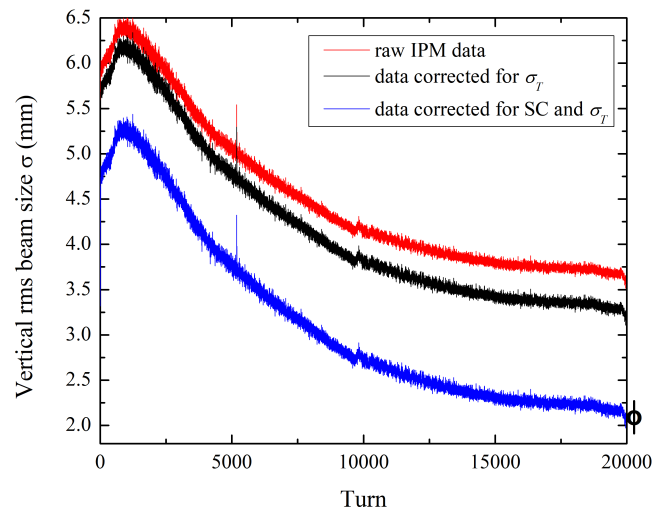

Figure 3: An example of reconstruction of vertical rms proton beam size in $33 \mathrm{~ms}$ (20000 turns) acceleration cycle of the Fermilab $8 \mathrm{GeV}$ Booster synchrotron with the total beam intensity of $N=4.6 \cdot 10^{12}$ : time dependence of the original IPM data (red), the data corrected for smearing effects (black) and the same data after additional correction for the space-charge expansion (blue). Black open circle with error bars at the left represents the measured Multi-Wires beam size of the extracted beam.

Knowing $\sigma_{T}, N$ and the IPM extracting field $V_{0} / D$ one can easily reverse Eq.(1) and find the original proton beam $\sigma_{0}$ from the measured and corrected $\sigma^{*}$, see, e.g., Ref. [22]. Fig.3 illustrates the result of such analysis for the measured profiles of the Booster beam with $N=4.62 \cdot 10^{12}$. There, the red curve is for the rms vertical beam size $\sigma_{m}(t)$ as measured by the IPM at each of 20 thousand turns of the Booster acceleration cycle; the black line represents the beam size after correction for the intensity independent smearing $\sigma^{*}$; and, finally, the true proton rms beam size $\sigma_{0}$ was reconstructed following the algorithm of Eqs. $(1,3)$ and is represented by the blue line. One can see that the overall beam size correction is about $15 \%$ early in the Booster acceleration cycle when the rms beam size is about $6 \mathrm{~mm}$. At the end of the cycle, with proton energy increased from $400 \mathrm{MeV}$ to $8 \mathrm{GeV}$, the correction is almost by a factor of two and accounting for the space-charge expansion is the most important. Also, one can see that the reconstructed IPM size at the end of the acceleration cycle matches well the extracted beam size measured by the Multi-Wires, as indicated by a black open circle with error bars at the right of Fig.3. 


\section{REFERENCES}

[1] P. Strehl, Beam Instrumentation and Diagnostics, vol. 120, Springer, 2006.

[2] F. Hornstra, W.H. DeLuca, in Proc. VI Conference on High Energy Acceleration, Cambridge, MA, 1967, pp. 374-377.

[3] H. Weisberg, et al, IEEE Trans. Nucl. Sci. 30, 2179 (1983).

[4] B. Hochadel, et al, NIM A, 343, 401 (1994).

[5] R. Anne, et al, NIM A, 329, 21 (1993).

[6] R. Connolly, et al, NIM A, 443, 2015 (2000).

[7] A. Jansson,et al, AIP Conf. Proc., 868, 159(2006).

[8] F. Benedetti, et al, EPJ Web of Conf., 225, 01009 (2020).

[9] R.S. Moore, A. Jansson, V. Shiltsev, J. Instrum., 4 (12), P12018 (2009).

[10] S. Levasseur, et al, J. Instrum., 12 (02), C02050 (2017).

[11] K. Wittenburg, arXiv:1303.6767.

[12] J. Amundson, et al, PRSTAB, 6, 102801 (2003).

[13] Workshop on Simulations, Design and Operational Experience of Ionisation Profile Monitors (GSI, May 21-24, 2017), 2017, https://indico.gsi.de/event/5366/

[14] R. Thern, Tech. Rep. BNL-39487 (1987).

[15] W.S. Graves, NIM A, 364, 19 (1995).

[16] V.Shiltsev NIM A, 986, 164744 (2021).

[17] M. Reiser, Theory and Design of Charged Particle Beams (John Wiley and Sons,2008).
[18] C. Bakker, E. Segre, Phys. Rev., 81, 489 (1951).

[19] D. Vilsmeier, M. Sapinski, R. Singh, Phys. Rev. Accel. Beams, 22, 052801 (2019).

[20] C. Dimopoulou, et al, J. Phys. B: At. Mol. Opt. Phys., 38, 593 (2005).

[21] J. Egberts, IFMIF-LIPAc Beam Diagnostics. Profiling and Loss Monitoring Systems (Ph.D. thesis, Universite Paris Sud - Paris X, 2012).

[22] J.Eldred, et al, PRAB, 24, 044001 (2021)

[23] see also J.Eldred et al, this Conf. WEXB08.

[24] E. Hubbard, et al., Booster Synchrotron, Tech. Rep. FERMILAB-TM-405, Fermilab, 1973, see also "Booster Rookie Book" at https://operations.fnal.gov/rookiebooks/BoosterV4.1.pdf.

[25] V. Shiltsev, Modern Phys. Lett. A, 32, 1730012 (2017).

[26] M. Convery, et al, Preprint FERMILAB-TM-2693 (FNAL, 2018).

[27] M. Minty, F. Zimmermann, Measurement and Control of Charged Particle Beams (Springer Nature, 2003).

[28] E. Bravin, arXiv:2005.07400.

[29] V. Lebedev, V. Shiltsev, Accelerator Physics at the Tevatron Collider (Springer, 2012)

[30] F. Roncarolo, Accuracy of the Transverse Emittance Measurements of the CERN Large Hadron Collider (Ph.D. thesis, Milan Polytechnic, 2005), Tech.Rep. CERN-THESIS-2005082. 